\title{
Cell Motility and Metastatic Potential Studies Based on Quantum Dot Imaging of Phagokinetic Tracks
}

Wolfgang J. Parak ${ }^{1,2}$, Rosanne Boudreau ${ }^{3}$, Mark A. Le Gros ${ }^{4}$, Daniele Gerion ${ }^{1,2}$, Daniela Zanchet $^{2}$, C. M. Micheel ${ }^{2}$, Shara C. Williams ${ }^{2}$, A. Paul Alivisatos ${ }^{1,2,}$, and Carolyn A. Larabell ${ }^{3,5, *}$

${ }^{1}$ Materials Science Division, Lawrence Berkeley National Laboratory, Berkeley, CA, USA

${ }^{2}$ Department of Chemistry, University of California, Berkeley, CA, USA

${ }^{3}$ Life Science Division, Lawrence Berkeley National Laboratory, Berkeley, CA, USA

${ }^{4}$ Genomics Division, Lawrence Berkeley National Laboratory, Berkeley, CA, USA

${ }_{*}^{5}$ Department of Anatomy, University of California, San Francisco, CA, USA

* corresponding authors

\begin{abstract}
A wide variety of eukaryotic cells are shown to engulf colloidal semiconductor nanocrystals, or quantum dots, when they migrate. Here we show that the uptake of the nanocrystals is directly correlated with the cell motility, by comparing in detail the motions of both cancerous and healthy human breast cancer cells, as well as several other cell types. The nanocrystals are more photochemically robust than organic dyes (which fade quickly) and do not perturb the cells. The ability to examine these behaviors in live cells over extended time periods, and to quantify changes in response to various molecular manipulations, provides a powerful tool for studying the processes of cell motility and migration - behaviors that are responsible for metastases of primary cancers.
\end{abstract}




\section{INTRODUCTION}

Motility and migration of cancerous cells are extremely detrimental properties that lead to metastases and formation of secondary tumors. Migration of cells and their metastatic potential are well known to be correlated, so that metastatic potential could be rapidly assayed by simply measuring motility ${ }^{1}$. Present methods for observing cell motility suffer from a number of practical limitations. In this paper we demonstrate that recent advances in semiconductor nanotechnology enable greatly improved cell motility assays, based upon the phagokinetic uptake of colloidal quantum dots. This is an important practical application of nanotechnology in biomedical science.

The most direct method for observing cell motility, time lapse videos of cells in culture $^{2}$, is restricted to measurements on just a few cells at a time, and therefore this approach is not widely used to make statistically significant studies of cell populations. Improved statistics can be obtained with the "scratched wound method" ${ }^{3,4}$, in which a region of the cell culture substrate is denuded of cells, and then the time scale for the filling of this "hole" is observed; unfortunately, the history of the cell migration paths are lost, and the analysis is complicated by subjective analysis of the complex and variable patterns of cell motion that lead to hole filling. A significant advance occurred with the development of the Boyden Chamber invasion assay, in which cells are seeded on one side of a membrane, and the rate of appearance of cells on the other side is monitored ${ }^{5,6}$. This method is by far the most widely used, yet it is extremely laborious; it requires that the cells be fixed, and thus destroyed, and it certainly does not allow for real time variation of the external conditions. 
Twenty-five years ago, Albrecht-Buehler proposed a method for studying cell motility based upon observation of "phagokinetic tracks" ${ }^{7,8}$. In the most general way, we can say that a phagokinetic track is generated when a cell passes over a layer of "markers", and ingests them, leaving behind a blank spot equal to the area the cell has traversed. In principle the method is very powerful, as it provides a rapid and automatic method for integrating cell motility while preserving the history of individual paths. Until now, the method has only been used in a limited way due to problems with the available markers. Luminescence could not be used for phagokinetic tracking, because organic dyes bleach too quickly. Instead, the only marker that has been used is submicron Au particles, which can be imaged optically in transmission, and these impose many limitations, so that the technique has not received widespread acceptance.

The Au particles must be large ( 0.15 microns) in order to be observable optically. Such large particles do not stick well to the substrate, and therefore have to be grown directly on the substrate in a process that yields highly inhomogeneous particle distributions. Since the particles are grown directly on the substrates, by pouring a hot (near boiling) aqueous solution onto the substrate, the range of usable substrates is limited. Further, due to the large size of the Au microcrystals, when a cell moves one diameter, it ingests a volume of $\mathrm{Au}$ corresponding to $\sim 1 \%$ of the total cell volume, there is a strong possibility that this perturbs the cell motility. This is particularly true for small cells, such as epithelial cells, which are of particular importance in studies of metastasis, and in neurons. The film homogeneity is poor, and the inter-particle distance is large, limiting the resolution. Finally, this method provides only one level of contrast, so that it is restricted to measurements in two dimensions and it cannot easily be coupled 
to information about chemical signals involved in cell motility. Here we demonstrate the use of nanometer size colloidal quantum dots as versatile and powerful markers for phagokinetic tracks with none of the limitations mentioned above.

Colloidal quantum dots are protein-sized crystals of inorganic semiconductors, initially developed for opto-electronic applications ${ }^{9,10}$. They are robust and efficient light emitters, with a wide range of potential applications in cell labeling ${ }^{11-14}$. When coated with a suitable solubilizing layer, such as silica, they are stable under physiological buffer conditions ${ }^{15}$. Until now, quantum dot based biological labeling experiments have been confined to static labeling. Here we first show that colloidal semiconductor nanocrystals are spontaneously ingested by a wide variety of cells, while remaining fully luminescent, thus enabling the tracking of dynamical phenomena inside cells over periods of weeks, and secondly we demonstrate that this phenomenon can be used for quantum dot based imaging of phagokinetic tracks. 


\section{RESUltS AND DISCUSSION}

In all the experiments, thin layers of colloidal semiconductor nanocrystals were deposited on collagen-coated tissue culture substrates, followed by seeding of cells. Two sizes of colloidal nanocrystals, $\mathrm{CdSe}(2.8 \mathrm{~nm}) / \mathrm{ZnS} / \mathrm{SiO}_{2}, 8 \mathrm{~nm}$ total diameter emitting at $554 \mathrm{~nm}$ and $\mathrm{CdSe}(4.1) / \mathrm{ZnS} / \mathrm{SiO}_{2}, 16 \mathrm{~nm}$ outer diameter emitting at $626 \mathrm{~nm}$ were used ${ }^{15}$. Two types of cell lines were examined in detail, human mammary epithelial tumor cells (MDA-MB-231) and non-tumor cells (MCF-10A), using both confocal fluorescence microscopy and multi-photon microscopy. Several other cell lines were also investigated, showing similar results.

The colloidal quantum dots were readily ingested by all the cell lines examined. For instance, as early as three hours after plating of the tumor cells on the nanocrystalcoated collagen, the tumor cells had engulfed numerous nanocrystals that are packaged in small clusters in the cytoplasm, presumably in vesicles (Fig. 1). Optical sections obtained with the confocal microscope verify that the nanocrystals are within the cell rather than on the cell surface (Fig. 2). A single $0.5 \mu \mathrm{m}$ thick optical section through the nanocrystalfilled vesicles in the MDA-MB-231 cells was obtained using two-photon excitation with a Ti-Sapphire laser, showing small packages of nanocrystals on the periphery of the large perinuclear vesicles; likely packages of nanocrystals prior to incorporation in the large storage vesicles (Fig. 2C). The large cytoplasmic volume occupied by the nanocrystalfilled vesicles becomes quite apparent in the 3-D reconstruction of these optical sections (Fig. 2D). 
These cells were also labeled with the dye FM 4-64, a lipophilic styryl dye frequently used to visualize vacuolar organelles and to study the endocytic pathway. The FM 4-64 rapidly faded during the first 10 minutes of data collection (one image collected every 20 seconds) due to significant photobleaching, a common characteristic of organic dyes (Fig. 1A-C). In contrast, no detectable decrease in the intensity of the green emitting nanocrystals was observed during the 16 minutes of imaging shown here (Fig. 1A-F), or after extended time periods of laser exposure. After uptake, small vesicles of nanocrystals were transported from the cell periphery to the perinuclear region. Transport of one such vesicle is shown in Figure 1 as it travels at $0.1 \mu \mathrm{m} / \mathrm{s}$ towards the nucleus, a velocity compatible with transport via microtubule motors. The growth of cells on a layer of red emitting nanocrystals generated identical results (not shown). The size of the nanocrystals had no effect on uptake, transport or storage of nanocrystals.

One week after growth on nanocrystal-coated collagen, all cells contained perinuclear nanocrystals (Figure 2). To determine whether nanocrystals were restricted to the cytoplasm, or had entered the nucleus, DAPI was added to visualize the chromosomes. Both DAPI and green emitting nanocrystals were examined simultaneously using two-photon excitation at $760 \mathrm{~nm}$ wavelength, providing thin optical sections through the nucleus and demonstrating that nanocrystals were excluded from the nucleus. There is also no evidence of degradation of these nanocrystals over time. Cells that were trypsanized, replated on collagen in the absence of nanocrystals, and examined three days later all contained large nanocrystal-filled vesicles around the nucleus. Since there were no nanocrystals on the extracellular matrix for new cells to engulf, the nanocrystals must be passed to daughter cells during cell division. 
The degree of nanocrystal uptake reflects the migratory behavior of the cells. MDA-MB-231 cells, which are a highly dedifferentiated, invasive mammary epithelial cancer cell line, voraciously engulf nanocrystals as they migrate. In doing so, they generate a region free of nanocrystals that clearly reveals their migratory pathways, as can be seen in cells that were cultured on nanocrystal-covered collagen for 24 hours (Fig. 4D-F). Such clearings are not seen around cells examined three hours after plating (Fig. 4A-C). In contrast, the MCF-10A cells, a highly differentiated, spontaneously immortalized, non-tumor mammary epithelial cell line that is not migratory, demonstrate an intact layer of nanocrystals around them with little or no evidence of motility or migration after 24 hours of culture on the nanocrystal-coated collagen (Fig. 4J-L). The layer of nanocrystals looks virtually identical to that seen around cells after three hours of growth on the nanocrystal-coated collagen (Fig. 4G-I). The behavior of these two cell types on nanocrystal-coated extracellular matrix is consistent with the behaviors characteristically associated with these cell types in the absence of nanocrystals; MDAMB-231 cells are very invasive and migratory and MCF-10A cells are non-invasive, relatively immotile cells ${ }^{16-18}$.

The nanocrystal-free zone around the MDA-MB-231 cells seen after 24 hours is created by the engulfment of the nanocrystals as the cells crawl around the dish, rather than the displacement or degradation of nanocrystals. This is clearly demonstrated in Figure 2, which shows optical sections of the cells obtained using confocal microscopy. Numerous large vesicles filled with nanocrystals are seen juxtaposed to the nucleus in the MDA-MB-231 tumor cells after 24 hours (Fig. 2B), whereas only a few, small foci containing nanocrystals can be seen in the MCF-10A cells (Fig. 2A). 
The uptake of nanocrystals likely reflects non-specific engulfment via a process of pino-, endo-, and/or phagocytosis of the surrounding matrix. Nanocrystals readily adhere to the cell surface, most likely due to interactions of the cell surface glycoproteins and glycolipids with the nanocrystal surface. Addition of a drop of nanocrystals to a dish of cells not previously exposed to nanocrystals results in the almost immediate coating of the entire cell surface with nanocrystals, clearly delineating all lamellipodia and filopodia (not shown). The same attraction probably occurs as cells encounter the nanocrystals as they crawl across the matrix, resulting in adherence of nanocrystals to the cell surface and subsequent engulfment. It is less likely that the nanocrystals trigger uptake via the process of receptor-mediated endocytosis, since they are non-biological, but it is possible that the associated matrix to which they are attached could trigger that process. Whatever the mechanism, cells engulf nanocrystals as they crawl, leaving behind a history of migratory movements and accumulating the evidence of their consumption in large perinuclear storage vesicles.

Colloidal quantum dot based phagokinetic tracking as demonstrated here, promises to be a versatile and powerful method of quantifying motility in a wide variety of circumstances. Because the preparation of the markers has been separated from the process of substrate priming, a wide variety of tissue culture substrates can be used, including growth on extracellular matrix substances. This approach generates a thicker, but more natural, environment compared to glass cover slips. The quantum dots are robust and do not photobleach, and yet they are small enough to be non-perturbative to the cells, and to be useful for phagokinetic tracking of small epithelial cells that are responsible for $90 \%$ of cancers. Since the detection is luminescence based, the tracks can 
be observed using confocal multi-photon microscopy. Dots with many emission colors can be prepared and bio-conjugated to a range of targeting molecules, so that it is possible to monitor cell motility and migration while simultaneously tracking specific proteins tagged with complementary fluorescent molecules (GFP, CFP, YFP, or BFP). Finally, it is of great interest to study motility in three dimensions in layered extracellular matrix media, and this is possible if the colloidal dots of different size are deposited in a vertical gradient, providing depth contrast. Quantum-dot based phagokinetic tracking can thus lead to a wide range of new methods for the quantification of cell motility and the rapid assessment of metastatic potential. 


\section{Experimental Protocol}

\section{$\underline{\text { Preparation of Nanocrystal Coated Cell Culture Substrates }}$}

A solution of Vitrogen $100(2.9 \mathrm{mg} / \mathrm{ml}$ Collagen 1 in $12 \mu \mathrm{M} \mathrm{HCl}$; Cohesion, Palo Alto, CA, USA) in PBS (1:44), at $4^{\circ} \mathrm{C}$, was added to cell culture/viewing dishes. These were either $35 \mathrm{~mm}$ plastic Petri dishes in which the bottom had been replaced with a \#1 glass coverslip or in Lab-Tek II chambered coverglass (4 or 8 well). These dishes were stored at $37^{\circ} \mathrm{C}$ for 1.5 hours. The excess liquid was then aspirated, nanocrystals were immediately added to the collagen, and the dishes were stored (covered with aluminum foil) in the hood until dry. Cells were added to the nanocrystal-coated viewing dishes at $10,000-20,000$ cells $/ \mathrm{cm}^{2}$ and stored in the incubator until needed.

\section{Confocal Microscopy}

For Fig. 1, cells were examined using the MRC-1024 laser scanning confocal microscope (Bio-Rad) with a Nikon Diaphot 200 microscope and a 60 X PlanApo oil immersion objective lens (1.4 numerical aperture). Z-series (successive images collected by stepping through the cell at $0.2-0.5 \mu \mathrm{m}$ intervals) were obtained to determine distribution of the nanocrystals and time-series (same optical section imaged at 30-second intervals over time) were obtained to examine the dynamics of nanocrystals in the cells. Nanocrystals were excited using a krypton-argon laser at $488 \mathrm{~nm}$. For Figs. 2-4, cells were examined using a Zeiss 510 NLO imaging system. Nanocrystals were excited using an argon laser at $488 \mathrm{~nm}$ (Figs 4 and 2A,B) or Ti-Sapphire laser at $760 \mathrm{~nm}$ (Figs. 2C,D and 4). 


\section{ACKNOWLEDGEMENTS}

D.Z. is grateful to FAPESP, proc. 99/08603-7, for financial support. C.M.M. is a Howard Hughes Medical Institute Predoctoral Fellow. This work was supported by NIH National Center for Research Resources, Grant Number 1 R01 RR-14891-01 through the U.S. Department of Energy under Contract No. DE-AC03-76SF00098, DOD Advanced Research Projects Agency (DARPA) under Grant No. N00014-99-1-0728 and by the Director, Office of Energy Research, Office of Science, Division of Materials Sciences, of the U.S. Department of Energy under Contract No. DE-AC03-76SF00098, by the United States Department of Energy Office of Biological and Environmental Research (contract DE-AC0376SF00098) and by the United States Department of Defense Award Number DAMD17-98-1-8182 (awarded to C.A.L.). 


\section{FIGURE LEGENDS}

Figure 1. Uptake and transport of quantum dots by breast tumor cells. Human mammary epithelial tumor cells (MDA-MB-231) were grown on collagen that had been coated with a thin layer of silanized, water-soluble, green fluorescent semiconductor nanocrystals. FM4-64 (\# T-3166, Molecular Probes, Eugene, OR, US) was added to the medium immediately prior to imaging. The FM4-64 was rapidly incorporated into the cell plasma membrane, internalized, and packaged in small vesicles (red). The cells also engulfed the nanocrystals and incorporated them into small, but initially separate vesicles (green). The cells were examined over time by collecting images every 30 seconds using confocal microscopy. The FM4-64 suffered from marked photobleaching and was barely detectable after $5 \mathrm{~min}$ (C) and not detectible after $10 \mathrm{~min}$ (D). In contrast, the nanocrystals demonstrated no photobleaching and actually become brighter upon

excitation as described previously ${ }^{15,19}$. Multiple nanocrystal-filled vesicles are seen in the cytoplasm; one such vesicle (blue arrow) can be seen traveling from the cell periphery (blue dot) toward the nucleus.

Figure 2. Quantum dots are stored in large vesicles. Human mammary epithelial tumor cells (MDA-MB-231) and non-tumor cells (MCF 10A) were grown on collagen gels coated with either green-emitting (8 nm-diameter) or red-emitting (16 nm-diameter) nanocrystals for 24 hours. Optical sections of live cells obtained using a $40 \mathrm{X}, 1.3 \mathrm{NA}$ lens determined that the nanocrystals are in the cell cytoplasm rather than coating the surface of the cells. (A) Non-tumor, relatively immotile cells contain a few small vesicles 
filled with red-emitting nanocrystals. The tumor cells have engulfed numerous greenemitting nanocrystals that have been packaged in large vesicles that are stored in the perinuclear region (B). The size of the nanocrystals had no influence on the ingestion. (C) An optical section obtained using two-photon excitation at $760 \mathrm{~nm}$ wavelength with a Tisapphire laser shows that the nanocrystals are not coating the surface of the vesicles; the vesicles are filled with nanocrystals. Several small clusters of nanocrystals are also seen approaching the large storage vesicles. (D) A 3D reconstruction of serial optical sections collected with the Ti-Sapphire laser at 0.5 micron intervals reveals the cytoplasmic volume occupied by the massive number of nanocrystals taken up by the tumor cells.

Figure 3. Quantum dots are stored in perinuclear vesicles. Large vesicles of greenemitting quantum dots are seen in the perinuclear region of human mammary epithelial tumor cells (MDA-MB-231) that had been cultured for 7 days. Simultaneous two-photon excitation (760 nm wavelength of a Ti-Sapphire laser) of the DNA label, DAPI, and the green-emitting nanocrystals demonstrates that the vesicles are very closely apposed to the nucleus.

Figure 4. Engulfment of nanocrystals reveals migratory paths of breast tumor cells. Human mammary epithelial tumor cells, MDA-MB-231 (A-F), and non-tumor cells, MCF 10A (G-L), were grown on collagen that had been coated with a thin layer of silanized, water-soluble, red fluorescent semiconductor nanocrystals. Images were collected with a confocal microscope using fluorescence detectors to examine the nanocrystals (B, E, H, K) and DIC (Differential Interference Contrast) to visualize the 
cells $(C, F, I, L)$; the merged images $(A, D, G, J)$ show the cells and the layer of nanocrystals. After 3 hours, the layer of nanocrystals beneath the tumor cells (B) and non-tumor cells $(\mathrm{H})$ looks fairly continuous. The cells can only be seen using DIC imaging alone $(\mathrm{C}, \mathrm{I})$ or in the merged fluorescent/DIC image $(\mathrm{A}, \mathrm{G})$ but not with fluorescence alone (B, H). After 24 hours large clearings in the nanocrystal layer are observed around the tumor cells (D, E), but not the non-tumor cells $(\mathrm{J}, \mathrm{K})$. The nanocrystal-filled tumor cells are also fluorescing brightly after 24 hours (E). The nontumor cells, in contrast, seen in DIC (L) cannot be detected with the fluorescent detector after 24 hours $(\mathrm{K})$. The images were collected at the optical section that best showed the layer of nanocrystals. Since some tumor cells are crawling into the underlying collagen after 24 hours, they are less distinct in the DIC image (F). Figs. A-C are a composite of several small images of adjacent regions on the dish. The arc of brighter fluorescence is due to photo brightening caused by illumination of that region to the mercury arc lamp. 


\section{REFERENCES}

1. Partin, A.W., Schoeniger, J.S., Mohler, J.L. \& Coffey, D.S. Fourier Analysis of Cell Motility: Correlation of Motility with Metastatic Potential. Proc. Natl. Acad. Sci. USA 86, 1254-1258 (1989).

2. Rajah, T.T., Abidi, S.M.A., Rambo, D.J., Dmytryk, J.J. \& Pento, J.T. The motile behavior of human breast cancer cells characterized by time-lapse videomicroscopy. In Vitro Cell. Dev. Biol. - Animal 34, 626-628 (1998).

3. Bürk, R.R. A Factor from a Transformed Cell Line that Affects Cell Migration. Proc. Natl. Acad. Sci. USA 70, 369-372 (1981).

4. Környei, Z., Czirok, A., Vicsek, T. \& Madarasz, E. Proliferative and migratory responses of astrocytes to in vitro injury. J. Neurosci. Res. 61, 421-429 (2000).

5. Boyden, S. The Chemotactic Effect of Mixtures of Antibody and Antigen on Polymorphonuclear Leucocytes. J. Exp. Med. 115, 453-466 (1962).

6. Yao, J., Harvath, L., Gilbert, D.L. \& Colton, C.A. Chemotaxis by a CNS Macrophage, the Microglia. Journal of Neuroscience Research 27, 36-42 (1990).

7. Albrecht-Buehler, G. Phagokinetic Tracks of 3T3 Cells: Parallels between the Orientation of Track Segments and of Cellular Structures Which Contain Actin or Tubulin. Cell 12, 333-339 (1977).

8. Albrecht-Buehler, G. The Phagokinetic Tracks of 3 T3 Cells. Cell 11, 395-404 (1977).

9. Bawendi, M.G., Steigerwald, M.L. \& Brus, L.E. The quantum mechanics of large semiconductor clusters ("quantum dots"). Annu. Rev. Phys. Chem. 41, 477-496 (1990). 
10. Alivisatos, A.P. Semiconductor Clusters, Nanocrystals, and Quantum Dots. Science 271, 933-937 (1996).

11. Chan, W.C.W. \& Nie, S. Quantum Dot Bioconjugates for Ultrasensitive Nonisotopic Detection. Science 281, 2016-2018 (1998).

12. Bruchez, M.J., Moronne, M., Gin, P., Weiss, S. \& Alivisatos, A.P. Semiconductor Nanocrystals as Fluorescent Biological Labels. Science 281, 2013-2016 (1998).

13. Dahan, M. et al. Time-gated biological imaging by use of colloidal quantum dots. Opt. Lett. 26, 825-827 (2001).

14. Pathak, S., Choi, S.-K., Arnheim, N. \& Thompson, M.E. Hydroxylated Quantum Dots as Luminescent Probes for in Situ Hybridization. J. Am. Chem. Soc. 123, 4103-4104 (2001).

15. Gerion, D. et al. Synthesis and Properties of Biocompatible Water-Soluble SilicaCoated CdSe/ZnS Semiconductor Quantum Dots. J. Phys. Chem. B in press (2001).

16. Plopper, G.E. et al. Migration of breast epithelial cells on Laminin-5: differential role of integrins in normal and transformed cell types. Breast Cancer Research and Treatment 51, 57-69 (1998).

17. Sliva, D., Mason, R., Xiao, H. \& English, D. Enhancement of the Migration of Metastatic Human Breast Cancer Cells by Phosphatidic Acid. Biochem. Biophys. Res. 268, 471-479 (2000).

18. Wong, N.C. et al. $\alpha$ Integrins mediate adhesion and migration of breast carcinoma cell lines. Clinical \& Experimental Metastasis 16, 50-61 (1998). 
19. Cordero, S.R., Carson, P.J., Estabrook, R.A., Strouse, G.F. \& Buratto, S.K. PhotoActivated Luminescence of CdSe Quantum Dot Monolayers. J. Phys. Chem. B 104, 12137-12142 (2000). 